\title{
Both Wnt signaling and epidermal stem cell-derived extracellular vesicles are involved in epidermal cell growth
}

\author{
Ling Leng ${ }^{1 * \dagger} \mathbb{D}$, Jie $\mathrm{Ma}^{2+}$, Luye $\mathrm{Lv}^{3+}$, Wenjuan Wang ${ }^{4}$, Dunqin Gao', Yunping Zhu ${ }^{2,5}$ and Zhihong Wu'
}

\begin{abstract}
Millions suffer from skin diseases. Functional interfollicular epidermal stem cells are needed in skin therapy or drug screening in vitro. We obtained functional interfollicular epidermal stem cells with intact stemness and cell junctions by treating them with Wnt3a. Moreover, epidermal stem cell-derived extracellular vesicles were useful in epidermal cell growth. Finally, functional epidermal 3D organoids with polarity were cultured using Wnt3a and the supernatant derived from interfollicular epidermal stem cells and fresh medium in a 1:1 ratio. These results provide novel directions for the improvement of skin organoids and their potential in clinical application.
\end{abstract}

Keywords: Epidermal stem cell, Extracellular vesicle, Organoids, Proteomics, Epidermal development, Extracellular matrix

\section{Background}

In mammals, several distinct cell populations of the skin comprise three basic layers-epidermal layer, dermal layer, and hypodermis or subcutaneous fat layer. The epidermis is the outermost layer, composed of stratified cell layers maintained by keratinocytes, including both stem cells and the mature cells in abundance. The basal layer of the epidermis has undifferentiated proliferative progenitor cells expressing keratins, including keratin 5 (K5) and keratin 14 (K14) [1]. These progenitors not only replenish the basal layer through self-renewal, but also progressively migrate upwards through the epidermis, differentiating to form mature keratinocytes expressing keratin 1 (K1), keratin 10 (K10), and involucrin, and finally the outer layers of the terminally differentiated, dead stratum corneum cells [2]. Skin homeostasis

\footnotetext{
*Correspondence: lengling@pumch.cn; zhenlinger@126.com

${ }^{+}$Ling Leng, Jie Ma and Luye Lv contributed equally to this work.

${ }^{1}$ Stem Cell and Regenerative Medicine Lab, Department of Medical Science Research Center, Translational Medicine Center, Peking Union Medical College Hospital, Peking Union Medical College and Chinese Academy of Medical Sciences, Beijing, China

Full list of author information is available at the end of the article
}

needs two types of epidermal stem cells (EpSCs) including interfollicular epidermal stem cells and hair follicle stem cells. After injury, the interfollicular epidermal stem cells replicate and differentiate to form the mature epidermis and regenerate hair follicles. Wnt signaling pathways are involved in the maintenance of both the two types of stem cells, and play an essential role during skin development $[2,3]$. Besides, the Wnt signaling pathways are important during wound healing [4] and regulate epidermal cell proliferation of skin EpSCs [5]. Canonical Wnt proteins result in the accumulation of unphosphorylated $\beta$-catenin protein stability by inhibiting GSK3 $\beta$ kinase [6], and $\beta$-catenin activates as a nuclear cofactor for the LEF1/TCF family of DNAbinding proteins to activate canonical Wnt downstream pathways [7].

Various junctional and cytoskeletal proteins, including cytoskeletal building blocks and associated cell-matrix proteins or intercellular junctions, control the regenerative capacity of the epidermis in maintaining the EpSCs, which is useful for epidermal morphogenesis and growth [8]. For example, interfollicular epidermal stem cells 
adhere on a thin layer of specialized extracellular matrix (ECM) proteins between the epidermal and dermal layers called the basement membrane (BM). The BM is composed of type IV and VII collagen, laminin, perlecan, nidogens, growth factors, and other ECM proteins [9]. ECM proteins in the BM connect the basal stem cells through various anchoring complexes, which coordinate the actin and microtubule network and establish cell polarity, so that the basal stem cells (EpSCs) differentiate and proliferate [2]. The major receptors of ECM proteins which interact with EpSCs are integrins [1,2]. Integrin subunit alpha 3 beta $1(\alpha 3 \beta 1)$ and alpha 6 beta 4 ( $\alpha 6 \beta 4)$ are the major epidermal integrins that bind the ligand laminin-5 [2]. The downregulation of integrins reduces proliferation in the basal epidermal layers $[10,11]$. Desmosomes are clustered transmembrane cadherins called desmogleins (DSGs) and desmocollins (DSCs) that bind to plakoglobin (PG) and plakophilins (PKPs) to form desmosomal complexes. Desmosomes directly link intercellular junctions and intermediate filament to regulate cellular growth and metabolism and support the cytoarchitecture of the epidermis $[12,13]$.

Millions suffer from skin diseases caused due to mutations or thermal and pressure injuries, such as epidermolysis bullosa (DB) or chronic diabetic ulcers [14]. With the development of the stem cell industry, functional seed cells are needed to treat patients with skin diseases or for drug screening. In addition, stem cellderived extracellular vesicles can mediate cell-cell communication through cell-specific mRNA or proteins. Stem cell-derived, cell-specific extracellular vesicles can regulate biological function and enhance tissue regeneration [15-18]. However, the role of EpSC-derived extracellular vesicles on EpSCs is unclear.

In this study, we established both 2D and 3D EpSC culture system using Wnt3a protein. In addition, we first described the proteomic characteristics of EpSC-derived extracellular vesicles and their important roles on epidermal development that may benefit clinical application or drug discovery in the future.

\section{Materials and methods}

\section{Human EpSC culture}

Human skin tissue samples were obtained from Chinese PLA General Hospital, and ethical approval and informed consent were obtained. The interfollicular epidermal stem cells were isolated as described $[19,20]$ with some modifications. Briefly, subcutaneous tissues and fat were removed and the tissues were digested with Dispase II $\left(2.5 \mathrm{mg} / \mathrm{mL}\right.$, Cat \#17150041, Gibco) at $37^{\circ} \mathrm{C}$ for $2 \mathrm{~h}$ to obtain the epidermal sheets. Thereafter, the samples were digested with pre-warmed Trypsin/EDTA $\left(0.25 \%\right.$, Cat \#25200072, Gibco) at $37^{\circ} \mathrm{C}$ for $10 \mathrm{~min}$, centrifuged at $1200 \times \mathrm{g}$ for $3 \mathrm{~min}$, and washed three times with PBS at $4{ }^{\circ} \mathrm{C}$. Subsequently, the cells were seeded onto 6-well plates, pre-coated with Matrigel (Cat \#354230, Corning). Advanced DMEM/F12 (Cat \#12634, Invitrogen) medium containing NEAA (1\%, Cat \#11140, Gibco), B-27 (2\%, Cat \#17504, GIBCO), GlutaMAX (1\%, Cat \#35050, Gibco), HEPES (1\%, Cat \#15630, Gibco), NAce (1 mM, Cat \#A9165, Sigma), hEGF $(50 \mathrm{ng} / \mathrm{mL}$, Cat \#236-EG, R\&D), A83-01 (2 $\mu$ M, Cat \#SML0788, Sigma), fosklin $(10 \mu \mathrm{M}$, Cat \#S2449, Selleck), and penicillin/ streptomycin (100 U/L, Cat \#15140163) with or without Wnt3a (50 ng/mL, Cat \#5036-WN R\&D) was prepared. The cells were incubated overnight and filmed using the Real-Time Cell History Recorder (JuLi stage, NanoEnTek Inc., Korea) inside the incubator. Images were taken every day using the bright channels. EpSCs (treated with or without Wnt3a or XAV939) were cultured in 96-well plates and counted daily for 5 days to perform the proliferation curve.

\section{D organoid culture}

For 3D cultures, the cells obtained from the epidermis were cultured on ultra-low attachment culture dishes, in advanced DMEM/F12 medium with sodium hyaluronate $(0.2 \%$, Cat \#H3506, Sigma) and Wnt3a for 5 days. The culture medium was partially replaced every alternate day, and XAV939 (1 $\mu \mathrm{M}$, Cat \#S1180, Selleck) was added, starting from day 5 . The organoids were harvested after 5 days of culture for the next experiment.

\section{Immunofluorescence}

Cells were fixed in $4 \%$ formaldehyde for $20 \mathrm{~min}$ and washed in PBS. Thereafter, the samples were treated with $0.25 \%$ Triton X-100 for 20 min, blocked in $10 \%$ serum for $1 \mathrm{~h}$ at $25^{\circ} \mathrm{C}$, and incubated with primary antibodies, such as COL17A1 (Cat \#ab184996, Abcam), K14 (Cat \#ab181595, Abcam), K10 (Cat \#ab9026, Abcam), PLEC (Cat \#sc-33,649, Proteintech), ITGB1 (Cat \#ab170874, Abcam), ITGA6 (Cat \#ab20142, Abcam), DSC2 (Cat \#60239-1-Ig, Proteintech), DSG1 (Cat \# 24587-1-AP, Proteintech), $\beta$-catenin (Cat \#8480S, Cell Signaling), P63 (Cat \#ab735, Abcam), CLDN1 (Cat \#13050-1-AP, Proteintech), and Ki67 (Cat \#ab15580, Abcam) overnight at $4{ }^{\circ} \mathrm{C}$. Thereafter, the cells were incubated with secondary antibodies for $1 \mathrm{~h}$ at $25^{\circ} \mathrm{C}$, counterstained with DAPI, and the samples were sealed with Fluoro-Gel for Photography. The pictures were taken at $\times 20$ or $\times 40$ magnification and analyzed using Volocity Demo $(\times 64)$.

\section{qRT-PCR analysis}

Total RNA was isolated using the RNeasy Mini Kit (Qiangen). Thereafter, the cDNA was synthesized using reverse transcriptase (ReverTra Ace ${ }^{\circ}$ qPCR RT Master Mix, Toyobo), according to the manufacturer's instructions. qRT-PCR was performed using the SYBR Green 
master mix (TOYOBO) on the Bio-Rad iQ5 Real-Time PCR detection system. Data were collected using the Bio-Rad CFX Manager software. Gene expression within a sample was normalized to GAPDH expression by the 2 $-\Delta \Delta \mathrm{Ct}$ method. The primer sequences for PCR are available in Supplementary Table S1.

\section{Extracellular vesicle isolation and identification}

The extracellular vesicles were isolated from the human epithelial stem cell (EpSC) culture supernatant using a differential centrifugation protocol $[21,22]$ with minor modifications. Briefly, the cells were grown in 10-15 $\mathrm{cm}^{2}$ flasks under normal culture conditions in media supplemented with $10-15 \%$ fetal bovine serum until they reached a confluency of $70-80 \%$. Thereafter, the cells were cultured for $>48 \mathrm{~h}$ in media supplemented with extracellular vesicle-free FBS (A27208, Gibco, FBS was depleted of bovine extracellular vesicles by ultracentrifugation at $110,000 \mathrm{~g}$ for $180 \mathrm{~min})$. The culture medium was collected and centrifuged at $300 \mathrm{~g}$ at $4{ }^{\circ} \mathrm{C}$ for $10 \mathrm{~min}$ and $2000 \mathrm{~g}$ at $4{ }^{\circ} \mathrm{C}$ for $20 \mathrm{~min}$ to remove dead cells. The supernatants were further centrifuged at $10,000 \mathrm{~g}$ for 30 min at $4{ }^{\circ} \mathrm{C}$ to eliminate contaminating cellular debris. The extracellular vesicles were pelleted from the final supernatants by ultracentrifugation for $70 \mathrm{~min}$ at $4{ }^{\circ} \mathrm{C}$ and 110,000 $g$ (Beckman SW32Ti rotor) using an SW32 Ti swinging bucket rotor (Beckman Coulter, Fullerton, CA). The pellets were washed in PBS to eliminate contaminating proteins. Another round of centrifugation was performed at high speed and the supernatants were discarded. The extracellular vesicles samples were used for transmission electron microscopy (TEM) and western blot analysis.

\section{TEM analysis}

The samples were washed in PBS and fixed overnight in $0.1 \%$ formalin and for $2 \mathrm{~h}$ in $2.5 \%$ glutaraldehyde. Thereafter, the samples were washed in PBS, dehydrated in ascending concentrations of ethanol, and air-dried. The ultrastructure of artificial skins was gold sputtered and mounted for imaging on a transmission electron microscope.

\section{Western blot analysis}

The extracellular vesicles were collected and homogenized on ice in RIPA buffer, supplemented with a protease inhibitor cocktail (04693116001, Roche). For immunoblotting, equal amounts $(20 \mu \mathrm{g}$ per lane) of extracellular vesicle lysates were separated on a 10\% SDS polyacrylamide gel. The samples were blocked with 5\% skim milk for $2 \mathrm{~h}$ and incubated with anti-CD9 (602321-Ig, Proteintech, 1:1000 dilution), K1 (Cat \#ab93652, Abcam, 1:1000 dilution), K14 (Cat \#ab181595, Abcam, 1: 20,000 dilution), ITGB1 (Cat \#ab170874, Abcam, 1:1000 dilution), ITGA6 (27189-1-AP, Proteintech, 1:1000 dilution), ERK1 (16443-1-AP, Proteintech, 1:1000 dilution), ECAD (Cat \#ab76055, Abcam, 1:1000 dilution), and ICAM1 (60299-1-Ig, Proteintech, 1:1000 dilution) overnight at $4{ }^{\circ} \mathrm{C}$. Thereafter, the blots were washed three times and incubated with secondary antibodies for 50 $\min$ at $37^{\circ} \mathrm{C}$. Subsequently, the blots were washed three times and the signals were detected using the Pierce ${ }^{\text {nx }}$ ECL Western Blotting Substrate (32,106, Thermo Fisher Scientific) and Millipore Immobilon ${ }^{\mathrm{Tm}}$ Western Chemiluminescent HRP Substrate (WBKLS0100, Millipore) at $25^{\circ} \mathrm{C}$.

\section{Mass spectrometry identification and data analysis}

The EpSC-derived extracellular vesicles were collected and digested using the protein lysis buffer. Thereafter, the protein mixtures were extracted, processed, and digested as described previously [23]. The peptide mixtures were analyzed using an Orbitrap Fusion Mass Spectrometer equipped with a nanoflow liquid chromatography system. All MS/MS raw files were processed using the MaxQuant software [24] (version 1.6.5.0), searched against the UniProt human database (https:// www.uniprot.org/, downloaded on April 2020). Protein quantification was performed according to the intensitybased absolute quantification method [25] as implemented in MaxQuant. The online tool DAVID (https:// david.ncifcrf.gov/) [26] was used to annotate the proteins according to biological processes, cellular components, and molecular functions. The Matrisome database [27] (http://matrisomeproject.mit.edu/) was used to annotate the extracellular matrix (ECM) proteins. Protein-protein interactions were retrieved from the online tool STRING [28] and the visualizations were built using Cytoscape [29] (version 3.7.2).

\section{Statistical analysis}

For all results, the statistical data are shown as means \pm S.E.M. Student's $t$ test was used to compare data between groups. ANOVA was used to compare three or more groups. Replicates used were biological replicates. Results were considered significant at $p \leq 0.05$. Statistical tests were carried out using the GraphPad Prism 6 (La Jolla, CA, USA) software for Apple Mac.

\section{Results}

\section{Establishment of human EpSC culture}

The epidermis was separated from human skin tissue with Dispase II digestion. Thereafter, the epidermal cells were isolated from the epidermis by trypsin digestion for the 2D culture on matrix gel (Fig. 1a). The adherent cells could grow clonally over time (Fig. 1b). EpSCs located in the basal layer of the human skin tissue [30], which expresses with stem markers (K14 and collagen type XVII 
A

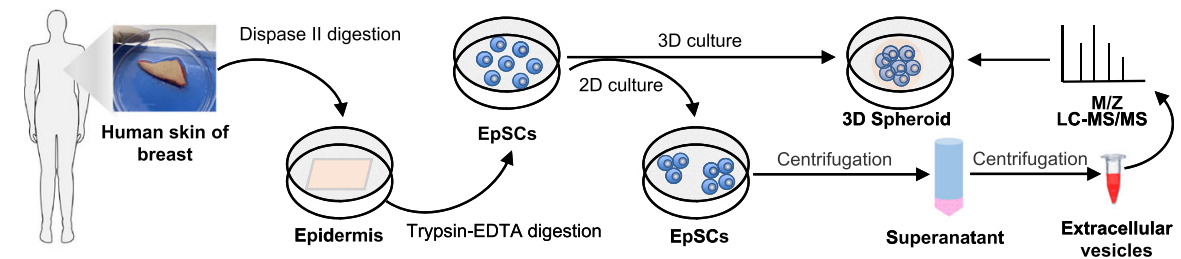

B
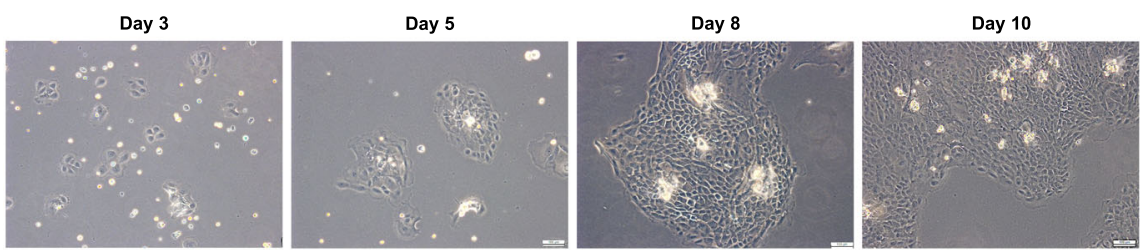

C
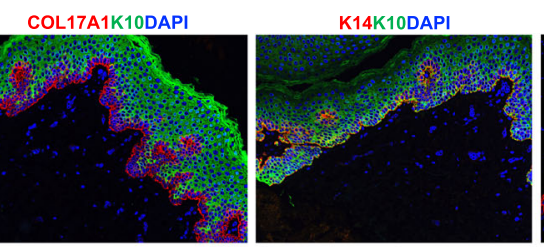

ITGB1Ki67DAPI

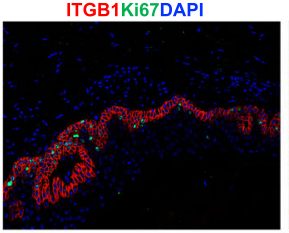

PLECITGA6DAPI

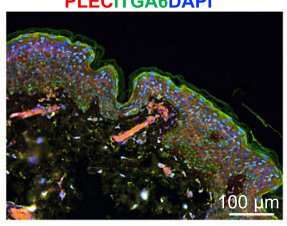

D

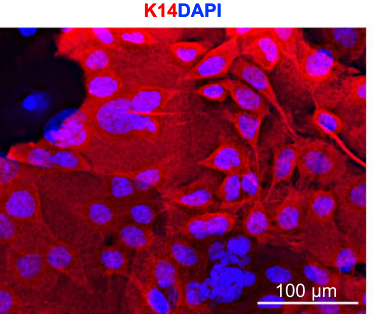

DSC2ITGB1DAPI

DSG1ITGA6DAPI
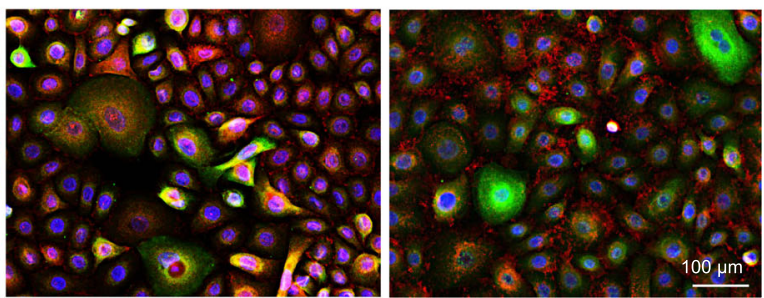

Fig. 1 Establishment of human epidermal stem cell (EpSC) Culture. a Schematic overview of tissue processing, EpSC culturing, and extracellular vesicle analysis. b Representative images show the generation of human EpSCs (scale bar $100 \mu \mathrm{m}$ ). c Immunostaining of COL17A1, K14, K10, ITGB1, Ki67, PLEC, and ITGA6 in human skin (scale bar $100 \mu \mathrm{m}$ ). d Immunostaining of K14, DSC2, ITGB1, DSG1, and ITGA6 in EpSCs (scale bar $100 \mu \mathrm{m})$. All biological experiments were repeated three times $(n=3$, biological replicates)

alpha 1 chain, COL17A1), adhesive receptor (integrin subunit beta 1, ITGB1), and hemidesmosome complexes (integrin subunit alpha 6, ITGA6, and plectin, PLEC) (Fig. 1c) [31]. Immunofluorescence staining showed that almost all clonally cultured epidermal cells expressed K14 (Fig. 1d). The EpSCs have capacity of adhesion since the cell-matrix junction markers (ITGA6) and cell-cell junction markers (desmosome, DSC2, and DSG1) were expressed in the EpSCs (Fig. 1d), indicating that the cultured EpSCs exhibit polarity. These results indicate that a 2D culture system of human EpSCs was successfully established.

\section{Wnt/ß-catenin signal promotes the stemness of EpSCs}

The Wnt signaling pathway is essential for epidermal stem cell self-renewal [32]. $\beta$-catenin, the key protein of the Wnt pathway, translocated into nucleus (Fig. 2a), indicating the expansion potential of the EpSCs. The EpSCs were treated with the $\beta$-catenin inhibitor (XAV939) to study the role of the Wnt pathway on fate determination. The results showed that more mature marker proteins (K10) were present in the XAV939 group compared to the control groups. However, the stemness marker (K14) was found to be upregulated with a little K10 after Wnt3a treatment (Fig. 2b). Proliferation markers (PCNA and Ki67) and proliferation curve revealed that the proliferation ability of EpSCs was enhanced after treated by Wnt3a (Fig. 2c-e). In addition, we found that Wnt3a-treated EpSCs could express more ECM-digesting matrix metallopeptidases (MMPs), such as $M M P 1, M M P 2, M M P 3$, $M M P 7, M M P 8$, and $M M P 19$, compared to the control group (Fig. 2f). ECM digestion is a secondary effect of cell proliferation [33], indicating that the proliferation ability of Wnt3a-treated EpSCs was higher. More ECM proteins, such as COL4A1, fibrillin 1 ( $F B N 1$ ), heparan sulfate proteoglycan 2 (HSPG2), alpha-2-macroglobulin $(A 2 M)$, and alpha-1-microglobulin/bikunin precursor $(A M B P)$, of the $\mathrm{BM}$, an important EpSC niche, were found in Wnt-treated EpSCs compared to the control group (Fig. 2g). On the other hand, the genes related to keratinocyte 


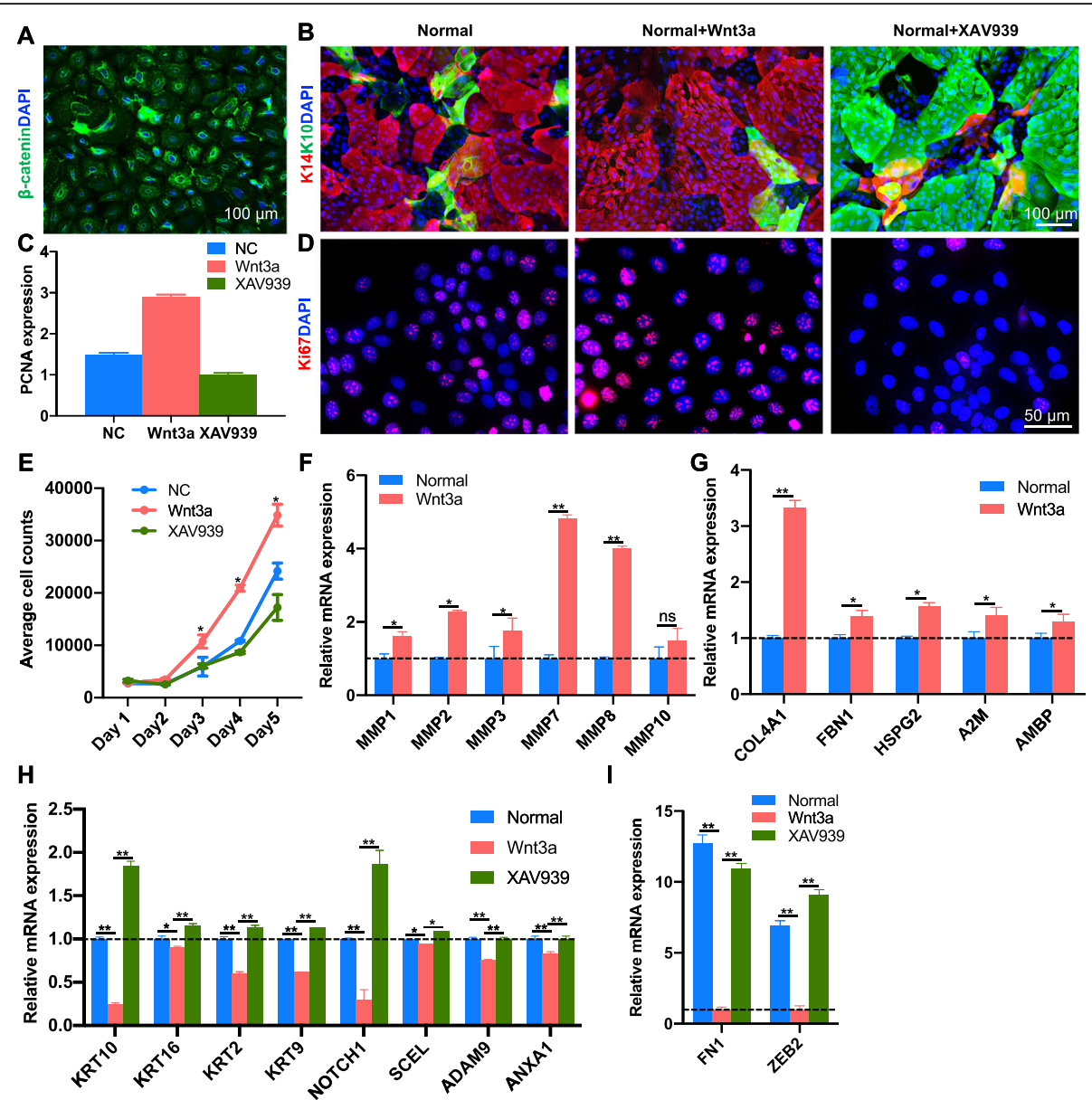

Fig. 2 Wnt/ $\beta$-catenin signaling promotes stemness and function of interfollicular epidermal stem cells (EpSCs). Immunostaining of $\beta$-catenin (a), K14 and K10 (b), and Ki67 (d) in EpSCs treated with Wnt3a or XAV939. c mRNA expression of PCNA in EpSCs treated with Wnt3a or XAV939. e Proliferation curve of EpSCs analysis in EpSCs treated with Wnt3a or XAV939. mRNA expression of MMPs (f), BM components (g), keratinocyte differentiation associated genes (h), and EMT associated genes (I), in EpSCs treated with Wnt3a or XAV939. All biological experiments were repeated three times $\left(n=3\right.$, biological replicates; data displayed as mean \pm SEM, $\left.{ }^{*} p<0.05,{ }^{* *} p<0.01\right)$

differentiation were downregulated in Wnt3a-treated EpSCs, and recovered in XAV939 group (Fig. 2h). The genes involved in epithelial mesenchymal transition (EMT), such as fibronectin 1 (FN1) and zinc finger E-box binding homeobox 2 (ZEB2), were downregulated in Wnt3a-treated EpSCs; however, they were upregulated in the XAV939 group (Fig. 2i), indicating the importance of the Wnt pathway on epidermization. These results indicate that activation of the $\mathrm{Wnt} / \beta$-catenin signaling pathway is necessary for determining EpSC fate.

\section{EpSC-derived extracellular vesicles mediate EpSC development}

Stem cell-derived extracellular vesicles are useful in regenerative medicine. To study the role of EpSC-derived extracellular vesicles in epidermal development, we collected extracellular vesicles from cultured EpSCs for proteomic analysis (Fig. 3a). Our results showed that EpSC-derived extracellular vesicles were enriched in cytoskeletal components, including the membrane (34.9\%), cytoskeleton (9.9\%), and keratin filament (3.3\%); cell adhesion molecules, including focal adhesion (21.8\%), hemidesmosome (0.9\%), cell-cell junction (5.7\%), and desmosome (1.2\%); lipid related proteins, including lipid particles (1.5\%), chylomicron $(0.9 \%)$, and very-low-density lipoprotein (0.9\%); melanosomes (8.4\%); and extracellular matrix (19.6\%) (Fig 3b, Supplementary Table S2). Tissue expression analysis showed that EpSC-derived extracellular vesicles were enriched in the epithelium and skin (Fig. 3c), indicating that most EpSC-derived extracellular vesicles may be epithelium specific and skin specific.

Biological process analysis showed that the proteins present in EpSC-derived extracellular vesicles were involved in epidermis development, including polarity, morphogenesis, and differentiation of epithelial cells, and the establishment of the skin barrier (Fig. 3d). In addition, processes of leukocyte movement and 


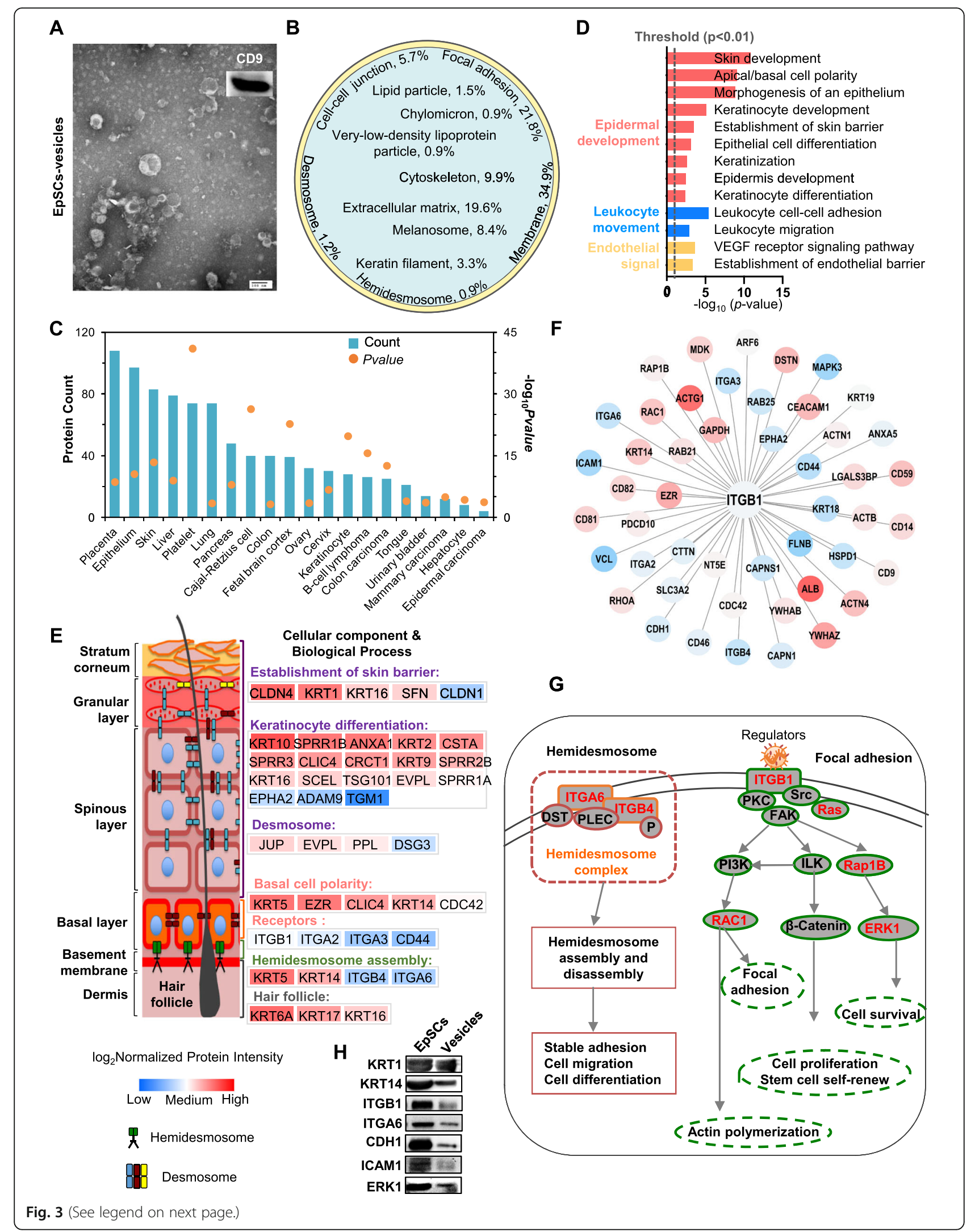


(See figure on previous page.)

Fig. 3 Epidermal stem cell (EpSC)-derived extracellular vesicles mediate EpSC development. a Transmission electron microscopy (TEM) and western blot analysis of EpSC-derived extracellular vesicles. $\mathbf{b}$ Schematic of percentage of cell components in EpSC-derived extracellular vesicles. Tissue expression (c) and biological process (d) analysis of EpSC-derived extracellular vesicles. e Schematic of cellular component and biological process in spatial function of the epidermis. The depth of the color indicates protein abundance according to $\log _{2}$ normalized protein intensity of EpSC-derived extracellular vesicles. $\mathbf{f}$ Interaction network between ITGB1 and other proteins of EpSC-derived extracellular vesicles. $\mathbf{g}$ Selected cellular pathways of hemidesmosome and focal adhesion. $\mathbf{h}$ Western blot analysis of KRT10, KRT14, ITGB1, ITGA6, ERK1, ICAM1, and ECAD in EpSCs and EpSC-derived vesicles. All biological experiments were repeated three times ( $n=3$, biological replicates)

endothelial signal were also enriched in EpSC-derived extracellular vesicles. To evaluate the role of EpSCderived extracellular vesicles in epidermal development, the functions of the EpSC-derived extracellular vesicles were analyzed according to the spatial function of skin. Our results showed that extracellular vesicle proteins were involved in functions associated with the dermal and epidermal layers of the skin, e.g., the hair follicles at the dermal papilla of the dermis (KRT6A, KRT17, and KRT16), receptors (ITGB1, ITGA2, ITGA3, and CD44) and polarity of the basal layer of epidermis (KRT5, EZR, CLIC4, KRT14, and CDC42), keratinocyte differentiation (KRT10, SPRR1B, ANXA1, KRT2, CSTA, SPRR3, CLIC4, CRCT1, KRT9, SPRR2B, KRT16, SCEL, TSG101, EVPL, SPRR1A, EPHA2, ADAM9, and TGM1), and establishment of barrier function of the epidermis (CLDN4, KRT1, KRT16, SFN, and CLDN1) (Fig. 3e). In addition, the important components of the dermisepidermis junction (DEJ), including KRT5, KRT14, ITGB4 and ITGB6, were also enriched in EpSC-derived extracellular vesicles (Fig. 3e).

ITGB1 is important for signal transduction in EpSCs. Interaction-network analysis showed that 48 proteins of EpSC-derived extracellular vesicles were associated with ITGB1 (Fig. 3f), indicating a role in signal transduction. Some were involved in the ITGB1-FAK pathways of EpSCs including members of the RAS superfamily (RAB25, RAP1B, and RAC1) and the MAP kinase family (MAPK3/ERK1) (Fig. 3f, g). Further, the stemness marker (K14), mature marker (K1), integrins (ITGB1 and ITGA6), cadherin (CDH1), adhesion molecule (ICAM1), and ERK1 were verified expressed in the vesicles (Fig. 3h). The results enabled us to evaluate whether EpSC-derived extracellular vesicles were associated with signaling pathways by activating ITGB1. Surprisingly, 36 soluble regulators of ECM were identified in EpSCderived extracellular vesicles according to the Matrisome database [27]. Some were involved in cell growth, such as cell proliferation (ANXA1, S100P, and FGFBP1), cell differentiation (ANXA5, S10017A, CRNN, S100A8, and MDK), and cell migration (ADAM9, S100A14, and S100A6) (Supplementary Table S3). For example, galectins (LGALS7), which are specifically expressed in the keratinocytes of the squamous epithelium, can directly function in epidermal cell growth. A protein group associated with the immune response and inflammation, such as ANXA5, S100A7A, IL1RN, IL18, and CRNN, was also identified. In addition, enzyme system related proteins, including proteases (CTSD and ADAM9) and protease inhibitors (CSTA, CSTB, SERPINB1, SERP INB2, SERPINB3, SERPINB5, and MUC20), were identified. Together, these results indicate that EpSC-derived extracellular vesicles can promote the function and development of EpSCs through signal transduction.

\section{Establishment of a functional human epidermal 3D organoid culture}

EpSCs derived from human tissues can mimic the cellular composition of their tissue-of-origin in 3D cultures called organoids [34]. To promote the function of 3D organoids, we designed an organoid culture system for the human epidermis using Wnt3a treatment for the first 5 days to maintain EpSC stemness (Fig. 4a). Immunofluorescence staining showed that the organoids expressed the stemness (K14, Fig. 4b) and proliferation (Ki67, Fig. 4c) markers at high levels. In addition, the transcription factor (P63) of EpSCs was expressed in the 3D culture (Fig. 4b). To retain the support of EpSCderived extracellular vesicles on organoid function, we maintained fresh medium in a 1:1 ratio. Results showed that cell-cell junction markers (DSG1 and CLDN1) and cell-matrix junction marker (ITGA6) were expressed well. Further, XAV939 was added to promote EpSC differentiation into mature epidermal cells at day 6 . Five days later, the mature marker (K10) was found in 3D organoids. These results indicate that we have successfully established a 3D culture system for EpSCs with the ability to proliferate and differentiate.

\section{Discussion}

The interaction between stem cells and stem cell niches is a hot topic in research. The EpSC niche of skin (BM) is important for its stemness in the natural state. We found that EpSCs isolated from the human skin and cultured with Wnt3a can express more components of the $\mathrm{BM}$, including COL4A1, FBN1, HEPG2, A2M, and AMBP (Fig. 2g), indicating that EpSCs can promote BM formation for tissue engineering or stem cell transplantation. Secondly, Wnt3a enhanced EpSCs' proliferation, and various MMPs, which enhance EpSCs proliferation 


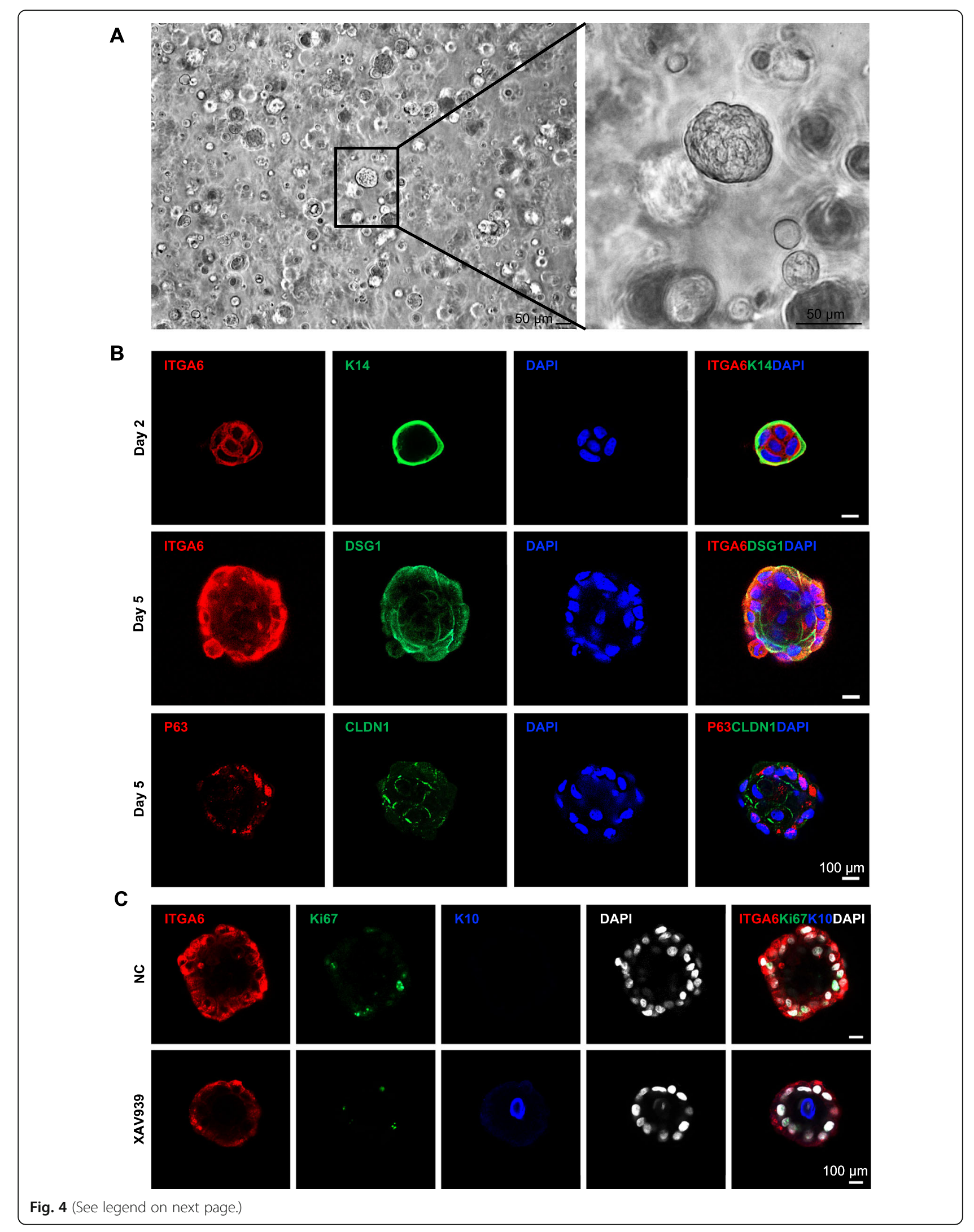


(See figure on previous page.)

Fig. 4 Establishment of functional human epidermal 3D organoid culture. a Representative images show the generation of human epidermal 3D organoids (scale bar $50 \mu \mathrm{m}$ ). b Immunostaining of ITGA6 and K14 at day 2 and ITGA6, DSG1, P63, and CLDN1 at day 5 (scale bar $100 \mu \mathrm{m})$. c Immunostaining of ITGA6, Ki67, and K10 at day 10 (scale bar $100 \mu \mathrm{m}$ ). All biological experiments were repeated three times $(n=3$,

biological replicates)

by degrading $\mathrm{BM}$ for self-renewal, were detected at a high level in Wnt3a-treated EpSCs. Thirdly, proteins (FN1 and ZEB2) associated with EMT decreased after Wnt3a treatment and increased after treatment with the inhibitor of $\beta$-catenin (XAV939) in EpSCs. EMT has been reported existing in stem cells, differentiated cells, and involved in many important physiological and pathological processes $[35,36]$. For example, skin injury needs re-epithelialization, which is considered as a partial EMT process [37], by the migration and proliferation of epidermal basal stem cells during wound healing [38]. In addition, the loss of epithelial polarity of epidermal cells in the EMT affects epidermal development and morphogenesis $[39,40]$. Therefore, EMT could be an important factor to consider for epithelium characterization. These results indicated that the $\mathrm{Wnt} / \beta$-catenin pathway was important for EpSC expansion and function. 3D organoids reflect near-physiological cellular composition and behavior than traditional 2D cultures [41]. In this study, we used Wnt3a protein, which is important for the self-renewal of EpSCs, as an additive to cultivate 3D organoids. Wnt3a was added in the early stages to improve the stemness and specific function of EpSCs in 3D culture.

The proteomic and functional characterization of EpSC-derived extracellular vesicles was also mapped. We found that EpSC-derived extracellular vesicles participate in the development of EpSCs, indicating the autocrine role of EpSCs. EpSC-derived extracellular vesicles were involved in each stage of development-from basal to mature layer-of interfollicular epidermal stem cells, including the hair follicles of the dermis, the EpSCs of the epidermal layer and the EpSC microenvironment, and the terminally differentiated epidermal layer. The epidermal development of the skin is related to the hemidesmosomes and focal adhesion. Hemidesmosomes are associated with the proliferation, migration, and differentiation of EpSCs through dynamic changes in assembly and disassembly to maintain homeostasis and wound repair. ITGB1 is a focal adhesion complex and regulates EpSC development and function through signal transduction. Our results indicated that the expression of hemidesmosome complexes and ITGB1 could be regulated by the form of EpSC-derived extracellular vesicles. In addition, 36 known ECM soluble factors were found to be secreted in the form of extracellular vesicles, possibly activating ITGB1 as growth factors. Many were directly involved in the maintenance and function of epidermal cells, further indicating the role of EpSC-derived extracellular vesicles in the functional culture of EpSCs. Finally, using the fluid secreted by EpSCs and the Wnt/ $\beta$-catenin inhibitor (XAV939), we obtained 3D organoids which were close to the epidermis, including basal and mature layers after 10 days.

\section{Conclusion}

In this study, we demonstrated the role of the Wnt pathway in enhancing the EpSCs' function through 2D/3D systems, as well as the potential role of EpSC-derived extracellular vesicles on skin development. These results provide novel directions for the improvement of skin organoids and tissue-engineered skin and stem cells for clinical transplantation and drug screening.

\section{Supplementary information}

Supplementary information accompanies this paper at https://doi.org/10. 1186/s13287-020-01933-y.

\section{Additional file 1: Supplementary Table S1. Details of primer} sequences used for PCR in this study.

Additional file 2: Supplementary Table S2. Proteome profile of epidermal stem cell (EpSC)-derived extracellular vesicles.

Additional file 3: Supplementary Table S3. List of extracellular matrix (ECM) proteins identified in epidermal stem cell (EpSC)-derived extracellular vesicles.

\section{Abbreviations}

BM: Basement membrane; DEJ: Dermis-epidermis junction; ECM: Extracellular matrix; EMT: Epithelial mesenchymal transition; EpSC: Epidermal stem cell; TEM: Transmission electron microscopy

\section{Acknowledgements}

We thank Dr. Shikun Zhang, Prof. Feng Gong, and all members from Department of Stem Cell and Regenerative Medicine Laboratory, Institute of Health Service and Transfusion Medicine, for their technical supports.

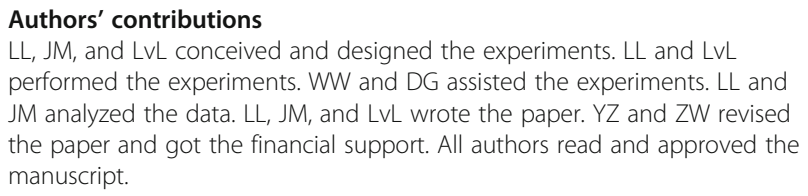

$L L, J M$, and $L V L$ conceived and designed the experiments. $L L$ and $L V L$ performed the experiments. WW and DG assisted the experiments. $L L$ and $J M$ analyzed the data. $L L, J M$, and $L V L$ wrote the paper. $Y Z$ and $Z W$ revised the paper and got the financial support. All authors read and approved the manuscript.

\section{Funding}

This work was supported by the National Key Research and Development Program of China (2016YFC0901701 and 2016YFB0201702), and the National Natural Science Foundation of China (31400701).

\section{Availability of data and materials}

The proteomics dataset supporting the conclusions of this article is available in the ProteomeXchange Consortium via the iProX [42] with the dataset identifier PXD020351 (http://proteomecentral.proteomexchange.org/cgi/ GetDataset?|D=PXD020351). 


\section{Ethics approval and consent to participate}

All human skin tissue samples were obtained from the Department of Dermatology, Chinese PLA General Hospital, after obtaining patients' informed consent. This study was approved by the Medical Ethics Committee of Chinese PLA General Hospital (NO. S2018-123-02).

\section{Consent for publication}

Not applicable.

\section{Competing interests}

The authors have declared that no competing interest exists.

\section{Author details}

${ }^{1}$ Stem Cell and Regenerative Medicine Lab, Department of Medical Science Research Center, Translational Medicine Center, Peking Union Medical College Hospital, Peking Union Medical College and Chinese Academy of Medical Sciences, Beijing, China. ${ }^{2}$ State Key Laboratory of Proteomics, Beijing Proteome Research Center, National Center for Protein Sciences (Beijing), Beijing Institute of Life Omics, Beijing, China. Institute of NBC Defense, Beijing, China. ${ }^{4}$ Department of Dermatology, Chinese PLA General Hospital, Beijing, China. ${ }^{5}$ Basic Medical School, Anhui Medical University, Hefei, Anhui, China.

Received: 3 August 2020 Revised: 29 August 2020

Accepted: 10 September 2020 Published online: 23 September 2020

\section{References}

1. Gonzales KAU, Fuchs E. Skin and its regenerative powers: an alliance between stem cells and their niche. Dev Cell. 2017;43(4):387-401.

2. Hsu YC, Li L, Fuchs E. Emerging interactions between skin stem cells and their niches. Nat Med. 2014;20(8):847-56.

3. Clevers H, Loh KM, Nusse R. Stem cell signaling. An integral program for tissue renewal and regeneration: Wnt signaling and stem cell control. Science. 2014;346(6205):1248012.

4. Miyoshi H, Ajima R, Luo CT, Yamaguchi TP, Stappenbeck TS. Wnt5a potentiates TGF- $\beta$ signaling to promote colonic crypt regeneration after tissue injury. Science. 2012;338(6103):108-13.

5. Myung PS, Takeo M, Ito M, Atit RP. Epithelial Wnt ligand secretion is required for adult hair follicle growth and regeneration. J Invest Dermatol. 2013;133(1):31-41.

6. Gordon MD, Nusse R. Wnt signaling: multiple pathways, multiple receptors, and multiple transcription factors. J Biol Chem. 2006;281(32):22429-33.

7. Fei Y, Xiao L, Doetschman T, Coffin DJ, Hurley MM. Fibroblast growth factor 2 stimulation of osteoblast differentiation and bone formation is mediated by modulation of the Wnt signaling pathway. J Biol Chem. 2011;286(47): 40575-83.

8. Hsu YC, Pasolli HA, Fuchs E. Dynamics between stem cells, niche, and progeny in the hair follicle. Cell. 2011;144(1):92-105.

9. Strong AL, Neumeister MW, Levi B. Stem cells and tissue engineering: regeneration of the skin and its contents. Clin Plast Surg. 2017;44(3):635-50.

10. Watt FM. Role of integrins in regulating epidermal adhesion, growth and differentiation. EMBO J. 2002;21(15):3919-26.

11. Margadant C, Charafeddine RA, Sonnenberg A. Unique and redundant functions of integrins in the epidermis. FASEB J. 2010;24(11):4133-52.

12. McAleer MA, Pohler E, Smith FJ, Wilson NJ, Cole C, MacGowan S, et al. Severe dermatitis, multiple allergies, and metabolic wasting syndrome caused by a novel mutation in the N-terminal plakin domain of desmoplakin. J Allergy Clin Immunol. 2015:136(5):1268-76.

13. Johnson JL, Najor NA, Green KJ. Desmosomes: regulators of cellular signaling and adhesion in epidermal health and disease. Cold Spring Harb Perspect Med. 2014;4(11):a015297.

14. Sen CK, Gordillo GM, Roy S, Kirsner R, Lambert L, Hunt TK, et al. Human skin wounds: a major and snowballing threat to public health and the economy. Wound Repair Regen. 2009;17(6):763-71.

15. Lai RC, Tan SS, Teh BJ, Sze SK, Arslan F, de Kleijn DP, et al. Proteolytic potential of the MSC exosome proteome: implications for an exosomemediated delivery of therapeutic proteasome. Int J Proteomics. 2012;2012: 971907.

16. Ching RC, Kingham PJ. The role of exosomes in peripheral nerve regeneration. Neural Regen Res. 2015;10(5):743-7.
17. Bauer N, Wilsch-Bräuninger M, Karbanová J, Fonseca AV, Strauss D, Freund $D$, et al. Haematopoietic stem cell differentiation promotes the release of prominin-1/CD133-containing membrane vesicles-a role of the endocyticexocytic pathway. EMBO Mol Med. 2011;3(7):398-409.

18. Wang Y, Yan W, Lu X, Qian C, Zhang J, Li P, et al. Overexpression of osteopontin induces angiogenesis of endothelial progenitor cells via the avß3/PI3K/AKT/eNOS/NO signaling pathway in glioma cells. Eur J Cell Biol. 2011;90(8):642-8.

19. Aasen T, Izpisúa Belmonte JC. Isolation and cultivation of human keratinocytes from skin or plucked hair for the generation of induced pluripotent stem cells. Nat Protoc. 2010;5(2):371-82.

20. Boonekamp KE, Kretzschmar K, Wiener DJ, Asra P, Derakhshan S, Puschhof J, et al. Long-term expansion and differentiation of adult murine epidermal stem cells in 3D organoid cultures. Proc Natl Acad Sci U S A. 2019;116(29): 14630-8.

21. Théry C, Amigorena S, Raposo G, Clayton A. Isolation and characterization of exosomes from cell culture supernatants and biological fluids. Curr Protoc Cell Biol 2006; Chapter 3:Unit 3.22.

22. Jia X, Chen J, Megger DA, Zhang X, Kozlowski M, Zhang L, et al. Label-free proteomic analysis of exosomes derived from inducible hepatitis B virusreplicating HepAD38 cell line. Mol Cell Proteomics. 2017;16(4 suppl 1):S144s160.

23. Wang W, Ma J, Sun X, Ba W, Meng X, Zhu Y, et al. A systems biology approach for defining the potential molecular framework of idiopathic hypereosinophilic syndrome with cutaneous involvement. Biochem Biophys Res Commun. 2020;524(3):567-74.

24. Cox J, Mann M. MaxQuant enables high peptide identification rates, individualized p.p.b.-range mass accuracies and proteome-wide protein quantification. Nat Biotechnol. 2008;26(12):1367-72.

25. Schwanhausser B, Busse D, Li N, Dittmar G, Schuchhardt J, Wolf J, et al. Global quantification of mammalian gene expression control. Nature. 2011; 473(7347):337-42.

26. Huang da W, Sherman BT, Lempicki RA. Bioinformatics enrichment tools: paths toward the comprehensive functional analysis of large gene lists. Nucleic Acids Res 2009; 37(1):1-13.

27. Naba A, Clauser KR, Ding H, Whittaker CA, Carr SA, Hynes RO. The extracellular matrix: tools and insights for the "omics" era. Matrix Biol. 2016; 49:10-24.

28. Szklarczyk D, Morris JH, Cook H, Kuhn M, Wyder S, Simonovic M, et al. The STRING database in 2017: quality-controlled protein-protein association networks, made broadly accessible. Nucleic Acids Res. 2017;45(D1):D362-8.

29. Shannon P, Markiel A, Ozier O, Baliga NS, Wang JT, Ramage D, et al. Cytoscape: a software environment for integrated models of biomolecular interaction networks. Genome Res. 2003;13(11):2498-504.

30. Fuchs EV, Coppock SM, Green H, Cleveland DW. Two distinct classes of keratin genes and their evolutionary significance. Cell. 1981;27(1 Pt 2):75-84.

31. Fuchs E, Nowak JA. Building epithelial tissues from skin stem cells. Cold Spring Harb Symp Quant Biol. 2008;73:333-50.

32. Kretzschmar K, Clevers H. Wnt/B-catenin signaling in adult mammalian epithelial stem cells. Dev Biol. 2017;428(2):273-82.

33. Sullivan WJ, Mullen PJ, Schmid EW, Flores A, Momcilovic M, Sharpley MS, et al. Extracellular matrix remodeling regulates glucose metabolism through TXNIP destabilization. Cell. 2018;175(1):117-32 e121.

34. Kretzschmar K, Clevers H. Organoids: modeling development and the stem cell niche in a dish. Dev Cell. 2016;38(6):590-600.

35. Chen T, You Y, Jiang H, Wang ZZ. Epithelial-mesenchymal transition (EMT): a biological process in the development, stem cell differentiation, and tumorigenesis. J Cell Physiol. 2017;232(12):3261-72.

36. Gros J, Tabin CJ. Vertebrate limb bud formation is initiated by localized epithelial-to-mesenchymal transition. Science. 2014;343(6176):1253-6.

37. Arnoux V, Côme C, Kusewitt DF, Hudson LG, Savagner P: Cutaneous wound reepithelialization. In: Rise and fall of epithelial phenotype. Molecular Biology Intelligence Unit. Boston: Springer; 2005: 111-34.

38. Park S, Gonzalez DG, Guirao B, Boucher JD, Cockburn K, Marsh ED, et al. Tissue-scale coordination of cellular behaviour promotes epidermal wound repair in live mice. Nat Cell Biol. 2017;19(2):155-63.

39. Box K, Joyce BW, Devenport D. Epithelial geometry regulates spindle orientation and progenitor fate during formation of the mammalian epidermis. Elife. 2019;8:e47102.

40. Cavallero S, Neves Granito R, Stockholm D, Azzolin P, Martin MT, Fortunel NO. Exposure of human skin organoids to low genotoxic stress can 
promote epithelial-to-mesenchymal transition in regenerating keratinocyte precursor cells. Cells. 2020;9(8):1912. .

41. Li M, Izpisua Belmonte JC. Organoids - preclinical models of human disease. N Engl J Med. 2019;380(6):569-79.

42. Ma J, Chen T, Wu S, Yang C, Bai M, Shu K, et al. iProX: an integrated proteome resource. Nucleic Acids Res. 2019;47(D1):D1211-7.

\section{Publisher's Note}

Springer Nature remains neutral with regard to jurisdictional claims in published maps and institutional affiliations.

Ready to submit your research? Choose BMC and benefit from:

- fast, convenient online submission

- thorough peer review by experienced researchers in your field

- rapid publication on acceptance

- support for research data, including large and complex data types

- gold Open Access which fosters wider collaboration and increased citations

- maximum visibility for your research: over $100 \mathrm{M}$ website views per year

At BMC, research is always in progress.

Learn more biomedcentral.com/submissions 